\title{
Metformin use and cardiovascular outcomes in patients with diabetes and chronic kidney disease: a nationwide cohort study
}

\author{
Min-Ho Kim ${ }^{1}$, Hyung Jung $\mathrm{Oh}^{2}$, Soon Hyo Kwon ${ }^{3,4}$, Jin Seok Jeon ${ }^{3,4}$, Hyunjin Noh ${ }^{3,4}$, Dong Cheol Han ${ }^{3,4}$, \\ Hyoungnae $\mathrm{Kim}^{3,4}$, Dong-Ryeol Ryu ${ }^{5}$ \\ ${ }^{1}$ Ewha Institute of Convergence Medicine, Ewha Womans University Mokdong Hospital, Seoul, Republic of Korea \\ ${ }^{2}$ Department of Nephrology, Sheikh Khalifa Specialty Hospital, Ras Al Khaimah, UAE \\ ${ }^{3}$ Department of Internal Medicine, Soonchunhyang University Seoul Hospital, Seoul, Republic of Korea \\ ${ }^{4}$ Hyonam Kidney Laboratory, Soonchunhyang University Seoul Hospital, Seoul, Republic of Korea \\ ${ }^{5}$ Department of Internal Medicine, Ewha Womans University School of Medicine, Seoul, Republic of Korea
}

Background: Metformin has recently been shown not to increase the risk of lactic acidosis in patients with chronic kidney disease (CKD). Thus, the criteria for metformin use in this population has expanded. However, the relationship between metformin use and clinical outcomes in CKD remains controversial.

Methods: This study considered data from 97,713 diabetes patients with an estimated glomerular filtration rate of <60 mL/min/1.73 $\mathrm{m}^{2}$. The primary outcome was major adverse cardiac and cerebrovascular events (MACCE), and the secondary outcomes were allcause mortality and incident end-stage renal disease (ESRD).

Results: Metformin users had a significantly higher risk of MACCE than non-users (hazard ratio [HR], 1.20; 95\% confidence interval [Cl], 1.14-1.26; $p<0.001)$. However, metformin users had a lower risk of all-cause mortality $(\mathrm{HR}, 0.78 ; 95 \% \mathrm{Cl}, 0.74-0.81 ; \mathrm{p}<$ $0.001)$ and ESRD (HR, 0.44; 95\% Cl, 0.42-0.47; $\mathrm{p}<0.001)$ during follow-up than non-users did. The relationships between metformin use and clinical outcomes remained consistent in propensity score matching analyses and subgroup analyses of patients with adequate adherence to anti-diabetes medication.

Conclusion: Treatment with metformin was associated with an increased risk of MACCE in patients with diabetes and CKD. However, metformin users had a lower risk of all-cause mortality and ESRD during follow-up than non-users did. Therefore, metformin needs to be carefully used in patients with CKD.

Keywords: Chronic kidney disease, Mortality, Metformin, Renal insufficiency

Received: November 15, 2020; Revised: May 31, 2021; Accepted: June 2, 2021

Editor: Soo Wan Kim, Chonnam National University, Gwangju, Republic of Korea

Correspondence: Hyoungnae Kim

Division of Nephrology, Department of Internal Medicine, Soonchunhyang University Seoul Hospital, 59 Daesagwan-ro, Yongsan-gu, Seoul 04401, Republic of Korea. E-mail: hkim@schmc.ac.kr

ORCID: https://orcid.org/0000-0002-5359-0214

Dong-Ryeol

Ryu Department of Internal Medicine, Ewha Womans University School of Medicine, 260 Gonghang-daero, Gangseo-gu, Seoul 07804, Republic of Korea. E-mail: dr6302@gmail.com

ORCID: https://orcid.org/0000-0002-5309-7606

Min Ho Kim and Hyung Jung Oh contributed equally to this work as co-first authors.

Copyright (C) 2021 by The Korean Society of Nephrology

(a) This is an Open Access article distributed under the terms of the Creative Commons Attribution Non-Commercial and No Derivatives License (http:// creativecommons.org/licenses/by-nc-nd/4.0/) which permits unrestricted non-commercial use, distribution of the material without any modifications, and reproduction in any medium, provided the original works properly cited. 


\section{Introduction}

Chronic kidney disease (CKD) is becoming a global health challenge [1]. Because it causes premature death and substantial healthcare costs, the increasing prevalence of CKD is a socioeconomic burden [2,3]. Diabetes mellitus (DM) is one of the most important causes of CKD in several countries [4], and the prevalence of DM in adults worldwide is expected to be $7.7 \%$ by 2030 [5]. Moreover, both DM and CKD are important risk factors for cardiovascular disease (CVD). The burden of CVD increases continuously as renal function declines [6], and individuals with both DM and CKD have an exceptionally high risk of CVD [7]. Therefore, proper DM treatment is an indispensable issue in CKD management.

Although new classes of anti-diabetes drugs have shown significant benefits in preventing CVD development [8,9], metformin is still the first-line drug for DM management $[10,11]$. Traditionally, metformin use has not been recommended for patients with CKD because of the risk of lactic acidosis. However, several clinical trials and observational studies have reported that the risk of fatal and nonfatal lactic acidosis did not increase with metformin use, even in patients with advanced CKD [12-14]. Accordingly, the U.S. Food and Drug Administration (FDA) of the United States expanded the criteria for metformin use. Nevertheless, prescribing metformin to advanced CKD patients, those whose estimated glomerular filtration rate (eGFR) is less than $30 \mathrm{~mL} / \mathrm{min} / 1.73 \mathrm{~m}^{2}$, remains controversial. Moreover, current clinical practice guidelines suggest diverse criteria for metformin use in patients with CKD [14], reflecting a lack of consensus about the relationship between metformin use and clinical outcomes in patients with CKD. Recent observational studies have shown inconsistent relationships between metformin use and CVD [15-17], allcause mortality $[13,18]$, and end-stage renal disease (ESRD) $[13,19,20]$. Clearly, physicians have reason to be confused about prescribing metformin to CKD patients.

Therefore, we evaluated the relationship between metformin use and the incidence of major adverse cardiac and cerebrovascular events (MACCE), all-cause mortality, and ESRD in CKD patients using data from a large Korean health screening cohort.

\section{Methods}

\section{Study population}

This retrospective observational study was conducted using cohort data from the National Health Insurance Service (NHIS) database. The NHIS data contain medical service claims, pharmacy claims, and health screening data for the whole population of the Republic of Korea. Detailed information about the NHIS database has been described previously [21]. The NHIS provides a national health screening program for adults aged $\geq 40$ years, and approximately three-quarters of all eligible Koreans participate in those health screenings every year. Among them, we selected 267,442 individuals who participated in a national health screening between 2009 and 2015, were prescribed anti-diabetes medications, and had an eGFR of $<60 \mathrm{~mL} /$ $\mathrm{min} / 1.73 \mathrm{~m}^{2}$. From them, we excluded individuals who had type 1 diabetes $(n=4,655)$, had ESRD requiring dialysis or kidney transplantation $(\mathrm{n}=583)$, had taken anti-diabetes medications for less than 90 days $(\mathrm{n}=163,913)$, or had experienced the clinical outcomes before taking anti-diabetes medication for 90 days $(n=1,064)$. However, we did not exclude patients with a history of CVD, such as myocardial infarction (MI), congestive heart failure, peripheral vascular disease, or stroke. Consequently, we included 97,713 patients in this study (Fig. 1). If patients underwent multiple health screenings, they were included at the earliest health screening that satisfied the inclusion criteria. Those patients were then followed up until December 31, 2019. This study was conducted in accordance with the Declaration of Helsinki. The study protocol was approved by the Institutional Review Board of Soonchunhyang University Seoul Hospital (No. 2019-06-014) with a waiver for informed consent.

\section{Data collection}

All health screening procedures met the internal and external quality control standards of the Korean Association of External Quality Assessment Service, as judged by detailed procedures described previously [22]. Briefly, data on lifestyle habits, including cigarette smoking and alcohol consumption, were obtained using a standardized questionnaire. The laboratory measurements and anthropometric 


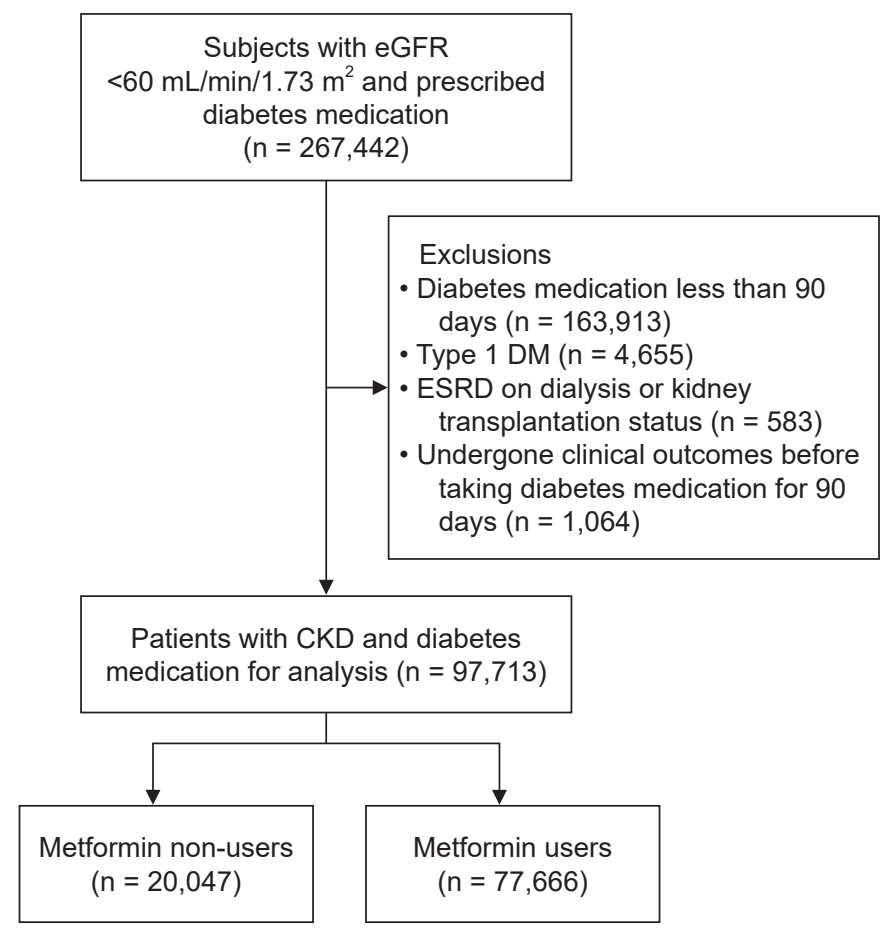

Figure 1. Flow chart for patient enrollment.

CKD, chronic kidney disease; DM, diabetes mellitus; eGFR, estimated glomerular filtration rate; ESRD, end-stage renal disease.

parameters were obtained by trained healthcare providers. Blood pressure was measured three times, and the average of the last two measurements was used. Blood samples were obtained after 8 hours of fasting, and eGFR was calculated using the CKD Epidemiology Collaboration equation [23]. Proteinuria was assessed by the dipstick test and was defined as $\geq 1+$. Medication history was obtained using the prescription database of the NHIS. Metformin, sulfonylureas, thiazolidinediones, a-glucosidase inhibitors, dipeptidyl peptidase-4 inhibitors, glucagon-like peptide-1 receptor agonists, sodium-glucose cotransporter-2 inhibitors, and insulin were considered anti-diabetes medications. In addition, we collected information about the use of aspirin, statins, and renin-angiotensin system blockade (RASB). Medical histories were obtained using the diagnosis codes in the NHIS database, which are based on the Korean Classification of Disease (KCD)-7. Medical histories were confirmed when patients visited an outpatient clinic at least twice or were hospitalized at least once due to disease before their first health screening day. The Charlson comorbidity index (CCI) was calculated using medical histories [24].

\section{Definition of metformin users}

In the Republic of Korea, prescriptions for chronic diseases, including DM, are generally given for 90 days. Therefore, we defined metformin users as individuals who had metformin prescriptions for at least 90 days after the health screening day. Metformin non-users were defined as diabetes patients who had prescriptions for other anti-diabetes medications (but not metformin) for the same period. Therefore, we defined the index date of study entry as the 90th day of the anti-diabetes medication prescription after the health screening day.

\section{Outcome assessment}

The primary outcome was MACCE, a composite of nonfatal coronary heart disease (CHD) (MI and unstable angina) (KCD code I20-I23) and nonfatal stroke (ischemic and hemorrhagic stroke) (I60-I63) requiring hospitalization for more than 2 days. The secondary outcomes were incident ESRD and all-cause mortality. Incident ESRD was defined as having prescription codes for hemodialysis or peritoneal dialysis for at least 90 days or having a prescription code for renal transplantation. Mortality data were linked with data prepared by Statistics Korea that record all deaths in the Republic of Korea. As a sensitivity analysis, we used cause of death data from Statistics Korea and defined cardiovascular mortality as death caused by MACCE.

\section{Statistical analysis}

Continuous variables are expressed as means \pm standard deviations, and categorical variables are presented as numbers and percentages. For comparisons between groups, Student t-test was used for continuous variables, and Pearson chi-square testing was used for categorical variables. The cumulative incidences of the outcomes were assessed using the Kaplan-Meier method. A multivariable analysis was conducted using a Cox proportional hazard regression model, and the results are expressed as hazard ratios (HRs) and $95 \%$ confidence intervals (CIs). As a sensitivity analysis, we conducted a cause-specific hazard regression analysis with mortality as a competing risk to evaluate the relationship between metformin use and MACCE or ESRD. We also used propensity score matching (PSM) analyses. The 
propensity scores were obtained using a logistic regression with the nearest neighbor technique, and metformin users were matched with metformin non-users in a ratio of 1:1. The logistic regression model was derived by adjusting for the following variables: age; sex; hypertension history; MI; congestive heart failure (CHF); peripheral vascular disease; stroke; cancer; use of aspirin, a statin, or RASB; body mass index (BMI); systolic blood pressure (SBP); fasting glucose; eGFR; serum total cholesterol; presence of proteinuria; smoking status with pack-years; and alcohol consumption. The PSM analyses were performed for the entire cohort and at each CKD stage. We also performed a sensitivity analysis using the subgroup with adequate adherence to anti-diabetes medication. Adequate adherence was defined as a proportion of days covered (PDC) with anti-diabetes medication greater than $80 \%$ of the entire follow-up period (PDC of metformin $\geq 80 \%$ for metformin users and PDC of other anti-diabetes medications (but not metformin) $\geq 80 \%$ for metformin non-users) [25]. Finally, we performed another PSM analysis in the subgroup whose PDC was $\geq 80 \%$. All statistical analyses were performed using SAS version 9.4 (SAS Institute Inc., Cary, NC, USA). A p-value of less than 0.05 was considered statistically significant.

\section{Results}

\section{Baseline characteristics}

Baseline characteristics according to metformin use are presented in Table 1. Among the 97,713 participants, 77,666 were classified as metformin users. The mean ages of the metformin users and non-users were $66.3 \pm 9.5$ and $66.0 \pm$ 8.9 years, respectively. Compared with the non-users, the metformin user group was younger, more female, and had a lower history of hypertension, MI, CHF, stroke, and cancer. Thus, the CCI among the metformin users was significantly lower than that in the metformin non-users group $(p<0.001)$. The use of aspirin was similar between groups, but metformin users took fewer statins and RASB than non-users. In addition, metformin users had lower BMI, SBP, and serum creatinine, but they had higher fasting glucose, eGFR, total and high-density lipoprotein cholesterol, and triglyceride levels than non-users. The metformin user group had fewer smokers and individuals with proteinuria but more heavy drinkers than the non-user group.

\section{Risk of major adverse cardiac and cerebrovascular events by metformin use status}

During a mean follow-up of 5.3 years, a total of 11,434 MACCE (11.7\%) occurred, for a corresponding incidence rate of 22.2 per 1,000 patient-years. The incidence rate of MACCE in metformin users was 22.5 per 1,000 patient-years, producing a significantly higher risk of MACCE than found in non-users (HR, 1.20; 95\% CI, 1.14-1.26; $\mathrm{p}<0.001$ ) (Table 2). When comparing the components of MACCE, the risk of CHD increased marginally (HR, 1.07 ; 95\% CI, 1.00-1.14; $\mathrm{p}=0.05$ ), but the risk of stroke increased significantly in metformin users (HR, 1.40; 95\% CI, 1.30-1.51; $\mathrm{p}<0.001$ ) compared with non-users. In the cause-specific hazard regression analysis with mortality as a competing risk, metformin users still had an increased risk of MACCE (HR, 1.36; 95\% CI, 1.28-1.44; p < 0.001). In the subgroups by CKD stage, the increased risk of MACCE among metformin users was observed in CKD stages 3a and $3 \mathrm{~b}$ but attenuated in CKD stages 4 and 5 (Supplementary Table 1, available online).

\section{Risk of all-cause mortality and end-stage renal disease by metformin use status}

During the follow-up period, the incidence rates of allcause mortality and ESRD were 24.1 and 7.6 per 1,000 patients-years, respectively. The risk of mortality during that period was significantly lower in metformin users (HR, 0.78; 95\% CI, 0.74-0.81; p 0.001) than in non-users (Table 3). The incidence of cardiovascular mortality was 3.9 and 2.8 per 1,000 patients-years in metformin non-users and users, respectively. Thus, risk of cardiovascular mortality was also significantly lower in metformin users (HR, 0.70; 95\% CI, 0.62-0.79; $\mathrm{p}$ < 0.001) (Supplementary Table 2, available online). However, due to their increased risk MACCE, metformin users had a higher risk than non-users of experiencing the composite cardiovascular outcome (HR, 1.16; 95\% CI, 1.10-1.22; $\mathrm{p}<0.001$ ), which was a composite of MACCE and cardiovascular mortality. In addition, the risk of ESRD was significantly lower in metformin users (HR, 0.44; 95\% CI, 0.42-0.47; $<$ 0.001) than in non-users (Table 3). In the cause-specific hazard regression analysis with mortality as a competing risk, metformin users still had a significantly lower risk of ESRD (HR, 0.48; 95\% CI, 0.44-0.52; p < 0.001) 
Table 1. Baseline characteristics of patients according to metformin use

\begin{tabular}{|c|c|c|c|}
\hline Characteristic & Metformin non-users & Metformin users & p-value \\
\hline No. of patients & 20,047 & 77,666 & \\
\hline Age (yr) & $66.3 \pm 9.5$ & $66.0 \pm 8.9$ & $<0.001$ \\
\hline Sex & & & $<0.001$ \\
\hline Male & $13,972(69.7)$ & $49,279(63.4)$ & \\
\hline Female & 6,075 (30.3) & $28,387(36.6)$ & \\
\hline \multicolumn{4}{|l|}{ Comorbid condition } \\
\hline Hypertension & $17,447(87.0)$ & $64,528(83.1)$ & $<0.001$ \\
\hline Myocardial infarction & $567(2.8)$ & $1,716(2.2)$ & $<0.001$ \\
\hline Heart failure & $1,072(5.3)$ & $2,844(3.7)$ & $<0.001$ \\
\hline Peripheral vascular disease & 2,383 (11.9) & $9,424(12.1)$ & 0.34 \\
\hline Stroke & $3,127(15.6)$ & 10,767 (13.9) & $<0.001$ \\
\hline Cancer & $1,153(5.8)$ & $3,419(4.4)$ & $<0.001$ \\
\hline \multicolumn{4}{|l|}{ Medication use } \\
\hline Aspirin & $7,787(38.8)$ & $30,020(38.7)$ & 0.62 \\
\hline Statin & $9,218(46.0)$ & $33,026(42.5)$ & $<0.001$ \\
\hline RASB & $14,852(74.1)$ & $53,834(69.3)$ & $<0.001$ \\
\hline Charlson comorbidity index & $2.6 \pm 1.6$ & $2.2 \pm 1.3$ & $<0.001$ \\
\hline \multicolumn{4}{|l|}{ Baseline measurement } \\
\hline Body mass index $\left(\mathrm{g} / \mathrm{m}^{2}\right)$ & $25.2 \pm 3.2$ & $25.1 \pm 3.2$ & $<0.001$ \\
\hline Systolic BP (mmHg) & $130.9 \pm 16.6$ & $129.5 \pm 16.0$ & $<0.001$ \\
\hline Diastolic BP (mmHg) & $77.1 \pm 10.3$ & $77.2 \pm 10.0$ & 0.51 \\
\hline Fasting glucose (mg/dL) & $131.8 \pm 47.0$ & $136.6 \pm 48.6$ & $<0.001$ \\
\hline Creatinine (mg/dL) & $1.7 \pm 2.1$ & $1.5 \pm 2.3$ & $<0.001$ \\
\hline $\operatorname{eGFR}\left(\mathrm{mL} / \mathrm{min} / 1.73 \mathrm{~m}^{2}\right)$ & $46.1 \pm 12.3$ & $50.7 \pm 9.6$ & $<0.001$ \\
\hline Total cholesterol (mg/dL) & $178.4 \pm 44.1$ & $180.4 \pm 43.1$ & $<0.001$ \\
\hline HDL cholesterol (mg/dL) & $48.1 \pm 18.6$ & $49.2 \pm 19.8$ & $<0.001$ \\
\hline LDL cholesterol (mg/dL) & $98.9 \pm 45.1$ & $99.2 \pm 73.9$ & 0.46 \\
\hline Triglycerides (mg/dL) & $163.8 \pm 114.9$ & $168.5 \pm 118.8$ & $<0.001$ \\
\hline Proteinuria $^{a}$ & 4,665 (23.3) & $10,773(13.9)$ & $<0.001$ \\
\hline Smoking status & & & $<0.001$ \\
\hline Non-smoker & $10,283(51.3)$ & $42,231(54.4)$ & \\
\hline Ex-smoker & $5,872(29.3)$ & $20,408(26.3)$ & \\
\hline Current smoker & $3,892(19.4)$ & $15,027(19.3)$ & \\
\hline Alcohol consumption (unit/wk) & & & $<0.001$ \\
\hline 0 & $8,565(42.7)$ & 33,695 (43.4) & \\
\hline $1-7$ & $5,733(28.6)$ & $21,080(27.1)$ & \\
\hline 8-14 & $2,392(11.9)$ & $9,172(11.8)$ & \\
\hline$\geq 15$ & $3,357(16.8)$ & $13,719(17.7)$ & \\
\hline
\end{tabular}

Data are expressed as number only, mean \pm standard deviation, or number (\%).

BP, blood pressure; eGFR, estimated glomerular filtration rate; HDL, high-density lipoprotein; LDL, low-density lipoprotein; RASB, renin-angiotensin system blockade.

${ }^{\text {a }}$ ipstick test, $\geq 1+$.

than non-users. In the subgroups of CKD stages, the lower risks of all-cause mortality and ESRD during follow-up in metformin users were consistently present in all CKD stages (Supplementary Table 3, available online).

\section{Risk of clinical outcomes by metformin use status after propensity score matching}

Because the patients' characteristics differed significantly 
Table 2. Risk of MACCE according to metformin use

\begin{tabular}{|c|c|c|c|c|c|c|c|}
\hline \multirow{2}{*}{ Outcome } & \multirow{2}{*}{ Metformin group } & \multirow{2}{*}{ Event, n (\%) } & \multirow{2}{*}{$\begin{array}{l}\text { Incidence (per } \\
1,000 \text { patient-yr) }\end{array}$} & \multicolumn{2}{|c|}{ Crude } & \multicolumn{2}{|c|}{ Adjusted $^{a}$} \\
\hline & & & & $\mathrm{HR}(95 \% \mathrm{Cl})$ & p-value & HR $(95 \% \mathrm{Cl})$ & p-value \\
\hline \multirow[t]{2}{*}{ MACCE } & Non-users & $2,082(10.4)$ & 21.1 & (Reference) & - & (Reference) & - \\
\hline & Users & $9,352(12.0)$ & 22.5 & 1.06 (1.01-1.11) & 0.021 & $1.20(1.14-1.26)$ & $<0.001$ \\
\hline \multirow[t]{2}{*}{$\mathrm{CHD}$} & Non-users & 1,261 (3.3) & 12.8 & (Reference) & - & (Reference) & - \\
\hline & Users & $4,983(6.4)$ & 12.0 & $0.93(0.88-0.99)$ & 0.028 & $1.07(1.00-1.14)$ & 0.05 \\
\hline \multirow[t]{2}{*}{ Stroke } & Non-users & $821(4.1)$ & 8.3 & (Reference) & - & (Reference) & - \\
\hline & Users & 4,369 (5.6) & 10.5 & 1.25 (1.16-1.35) & $<0.001$ & $1.40(1.30-1.51)$ & $<0.001$ \\
\hline
\end{tabular}

$\mathrm{CHD}$, coronary heart disease; $\mathrm{Cl}$, confidence interval; $\mathrm{HR}$, hazard ratio; MACCE, major adverse cardiac and cerebrovascular events.

${ }^{a}$ Adjusted for age, sex, smoking, alcohol consumption, history of hypertension, myocardial infarction, congestive heart failure, peripheral vascular disease, stroke, cancer, body mass index, systolic blood pressure, fasting glucose, estimated glomerular filtration rate, total cholesterol, presence of proteinuria, and use of aspirin, a statin, or renin-angiotensin system blockade.

Table 3. Risk of all-cause mortality and ESRD according to metformin use

\begin{tabular}{|c|c|c|c|c|c|c|c|}
\hline \multirow{2}{*}{ Outcome } & \multirow{2}{*}{$\begin{array}{l}\text { Metformin } \\
\text { group }\end{array}$} & \multirow{2}{*}{ Event, n (\%) } & \multirow{2}{*}{$\begin{array}{l}\text { Incidence (per } \\
1,000 \text { patient-yr) }\end{array}$} & \multicolumn{2}{|c|}{ Crude } & \multicolumn{2}{|c|}{ Adjusted $^{a}$} \\
\hline & & & & $\mathrm{HR}(95 \% \mathrm{Cl})$ & p-value & $\mathrm{HR}(95 \% \mathrm{Cl})$ & $p$-value \\
\hline \multirow[t]{2}{*}{ All-cause mortality } & Non-users & 3,370 (16.8) & 32.5 & (Reference) & - & (Reference) & - \\
\hline & Users & $9,830(12.7)$ & 22.2 & $0.66(0.63-0.68)$ & $<0.001$ & $0.78(0.74-0.81)$ & $<0.001$ \\
\hline \multirow[t]{2}{*}{ ESRD } & Non-users & 1,966 (9.8) & 20.1 & (Reference) & - & (Reference) & - \\
\hline & Users & $2,116(2.7)$ & 4.8 & $0.23(0.22-0.25)$ & $<0.001$ & $0.44(0.42-0.47)$ & $<0.001$ \\
\hline
\end{tabular}

$\mathrm{Cl}$, confidence interval; ESRD, end-stage renal disease; HR, hazard ratio.

${ }^{a}$ Adjusted for age, sex, smoking, alcohol consumption, history of hypertension, myocardial infarction, congestive heart failure, peripheral vascular disease, stroke, cancer, body mass index, systolic blood pressure, fasting glucose, estimated glomerular filtration rate, total cholesterol, presence of proteinuria, and use of aspirin, a statin, or renin-angiotensin system blockade.
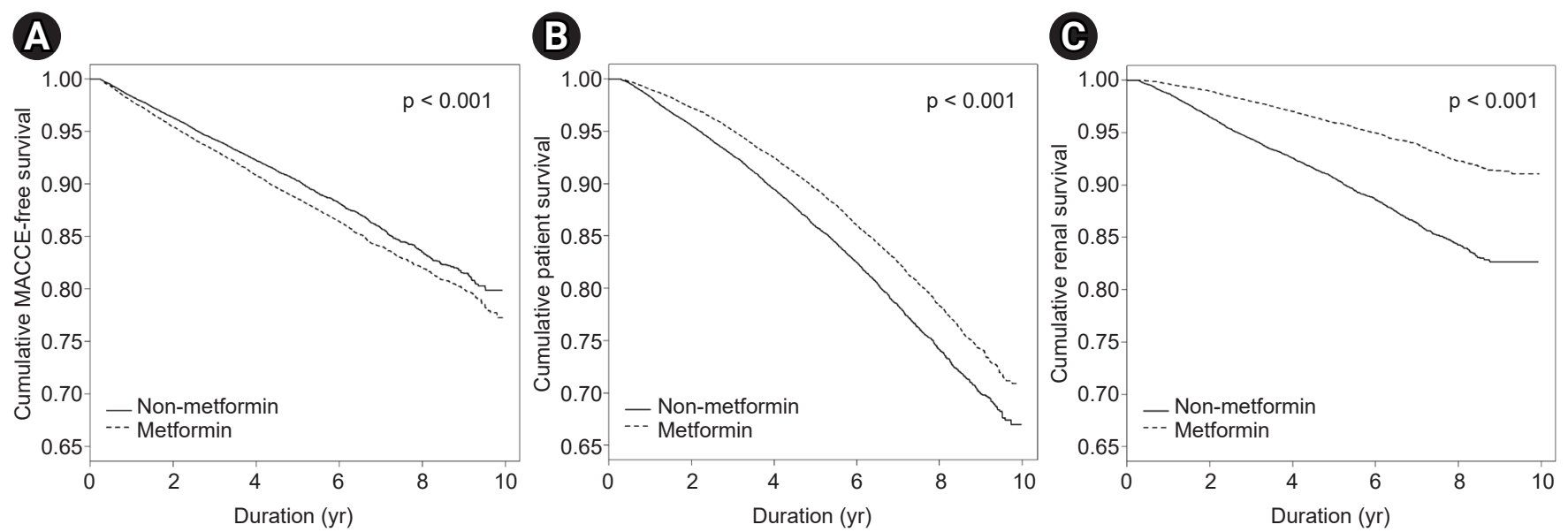

Figure 2. Cumulative event-free survival according to use of metformin in patients with chronic kidney disease and taking diabetes medication, after propensity score matching. (A) Major adverse cardiac and cerebrovascular events (MACCE), (B) all-cause mortality, and (C) end-stage renal disease (ESRD).

between groups, we performed PSM between metformin users and non-users in a 1:1 ratio. The baseline characteristics after PSM are presented in Supplementary Table 4 (available online). After PSM, the cumulative MACCE-free survival was significantly lower in metformin users than in non-users ( $\mathrm{p}<0.001$ ) (Fig. 2). Meanwhile, metformin users had significantly higher cumulative survival and ESRD-free survival than non-users $(\mathrm{p}<0.001$, all). In the multivariable analysis, the HR of MACCE for metformin use was 1.15 (95\% CI, 1.09-1.22) (Table 4). When we performed a PSM anal- 
ysis in each CKD stage, an increased risk of MACCE was observed only in CKD stage 3a; the risk was attenuated in advanced stages. In addition, the HRs of all-cause mortality and ESRD with metformin use were 0.76 (95\% CI, 0.73-0.80) and 0.45 (95\% CI, 0.42-0.48), respectively, and the benefits of metformin use for delaying death and preventing ESRD were seen in all CKD stages.

Risk of clinical outcomes by metformin use status in the subgroups with adequate adherence

A further sensitivity analysis was performed in the subgroup of patients with adequate adherence to anti-diabetes medication. The metformin users in this subgroup still had a significantly increased risk of MACCE (HR, 1.66; 95\% CI, 1.571.76; $\mathrm{p}<0.001$ ), and that relationship was observed in CKD stage 3 , but not in stages 4 and 5 (Table 5). Furthermore, the HR for all-cause mortality during follow-up was still significantly lower for metformin users in this subgroup (HR, 0.88; 95\% CI, 0.83-0.93; $\mathrm{p}<0.001$ ), but the survival benefit from metformin use was attenuated in patients with eGFR of $<45$ $\mathrm{mL} / \mathrm{min} / 1.73 \mathrm{~m}^{2}$. Meanwhile, the HR for ESRD in metformin users was 0.29 (95\% CI, 0.26-0.33) and consistently lower in metformin users than in non-users in all CKD stages.

Risk of clinical outcomes by metformin use status after PSM in the subgroups with adequate adherence

Finally, we conducted PSM in the subgroup of patients with adequate adherence to anti-diabetes medication. We found that metformin use still carried a significantly increased risk of MACCE (HR, 1.65; 95\% CI, 1.55-1.75; p < 0.001) (Table 6). When PSM was conducted for each CKD stage, that relationship was attenuated in the higher CKD stages, but it remained significant. Meanwhile, the survival benefit from metformin use also remained (HR, 0.89; 95\% CI, 0.84$0.95 ; \mathrm{p}<0.001$ ), but it disappeared in patients with eGFR of $<45 \mathrm{~mL} / \mathrm{min} / 1.73 \mathrm{~m}^{2}$. The risk of ESRD was significantly lower in metformin users (HR, 0.30; 95\% CI, 0.26-0.33; $\mathrm{p}<$ 0.001 ), and that benefit was observed in all CKD stages.

\section{Discussion}

In this study, we found that metformin use was associated with a significantly increased risk of MACCE in patients

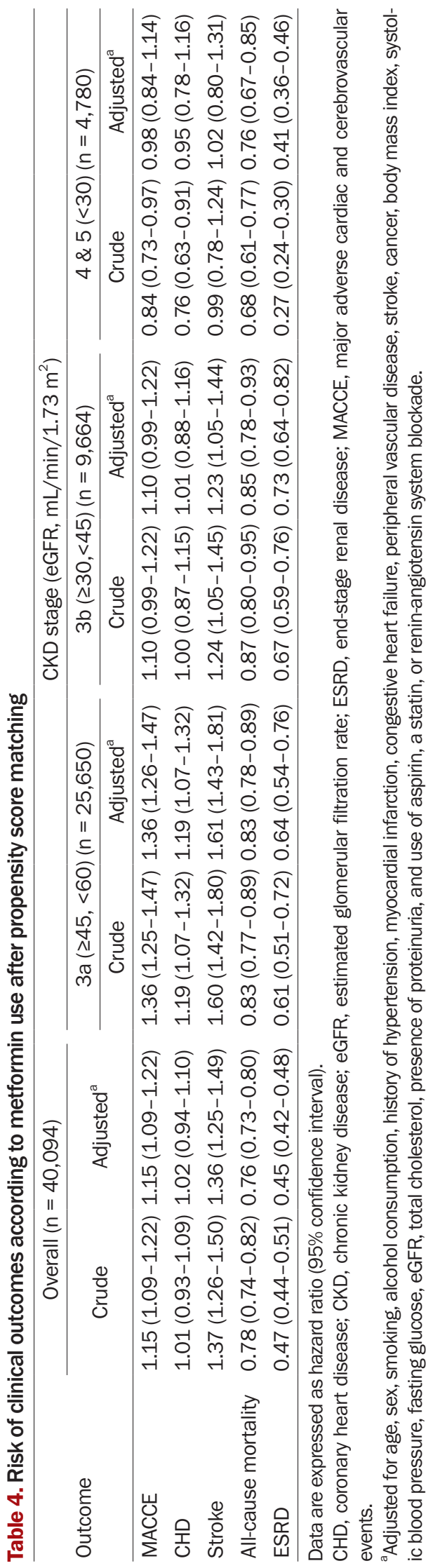



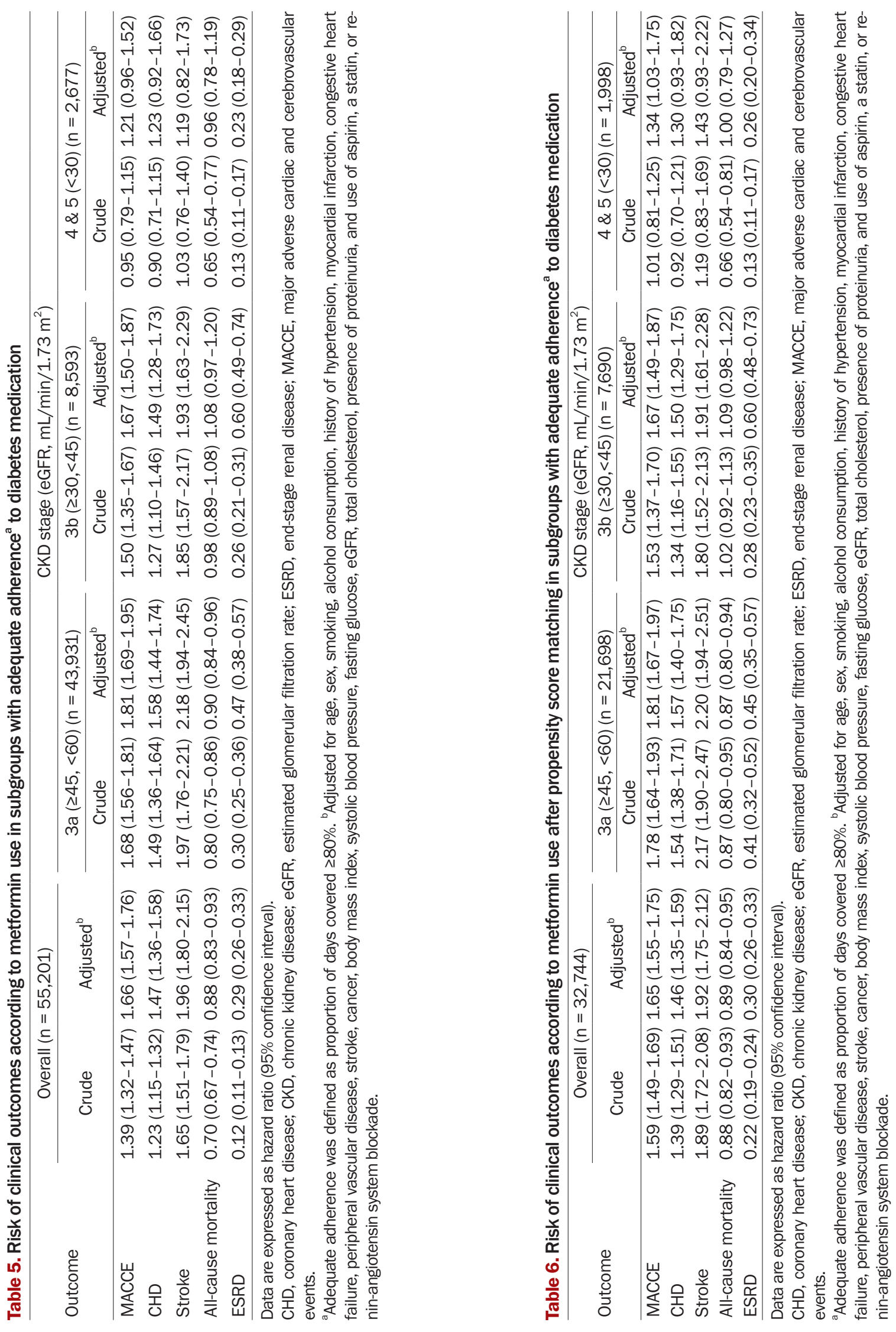
with CKD. However, metformin users had significantly lower risks of all-cause mortality and ESRD during follow-up than non-users did. These relationships were consistent in the subgroups made using a PSM analysis, adequate adherence to anti-diabetes medication, and both together. The higher risk of MACCE and lower risk of mortality during follow-up in metformin users were mitigated at advanced CKD stages; however, the benefit for ESRD was consistently shown in all CKD stages.

Although several new classes of anti-diabetes drugs have been released, metformin is still the first-line drug for DM management. In Korea, metformin is even the most commonly used anti-diabetes medication in patients with early CKD [26]. Beyond glycemic control, it has been reported that metformin has pleiotropic effects that control lipids, body weight, and blood pressure [27,28]. Nevertheless, previous studies have raised uncertainties about the effectiveness of metformin in reducing cardiovascular outcomes in the general population [29-31]. In 2016, the U.S. FDA allowed metformin use in patients with CKD stage 3, and the Korean Ministry of Food and Drug Safety followed the U.S. FDA in that ruling in 2019. However, the relationship between metformin use and cardiovascular outcomes has remained inconclusive in patients with CKD.

In a Swedish study of 51,675 patients with type 2 diabetes, treatment with metformin showed a CVD benefit when compared with insulin treatment, but not when compared with other oral hypoglycemic agents [17]. In addition, those researchers found no significant relationship between metformin use and the risk of any CVD in the subgroup of CKD stage 3 patients. Charytan et al. [15] reported that metformin users in CKD stage 3, but not those in CKD stages 4 and 5, had a lower risk of cardiovascular death than non-users. A recent study by Roumie et al. [16] reported that metformin monotherapy was associated with a lower risk of MAACE than sulfonylurea monotherapy in patients with CKD. In contrast, we found that metformin users with CKD faced a significantly increased risk of MACCE. Some patient characteristics differed between those previous studies and this study. First, the mean BMI in the studies conducted by Charytan et al. [15] and Roumie et al. [16] was greater than $30 \mathrm{~kg} / \mathrm{m}^{2}$, which is higher than that in our study population $\left(25.1 \mathrm{~kg} / \mathrm{m}^{2}\right)$. A previous study found that metformin is efficacious in lowering serum glucose regardless of BMI, but the weight reduction effect was observed only in obese patients [32]. Therefore, the cardiovascular protective effect of metformin found in previous studies might have been caused by weight reduction, which would not be expected to apply to our study population. Second, the patients in our study had a lower incidence of CHD and higher incidence of stroke than the patients in previous studies. In particular, the high risk of MACCE we found was largely caused by a high risk of stroke in metformin users, and the HR of stroke was higher than that of CHD in all our analyses. Activating the AMP-activated protein kinase (AMPK) pathway is one of the main molecular mechanisms of metformin [33]. However, AMPK activation in the brain is remarkably increased after an ischemic injury [34]. Moreover, some experimental studies have reported that metformin treatment and subsequent AMPK activation can aggravate acute cerebral infarction [35]. Importantly, at a concentration higher than the therapeutic dose, metformin can further activate the AMPK pathway [33,36]. Because metformin is eliminated by the kidney, its concentration can increase when renal function declines [37]. Therefore, a previous observational study found that metformin use was associated with an increased risk of stroke in ESRD patients on dialysis [38]. Accordingly, although we could not examine the specific doses of metformin used, high concentrations of it might have produced the high incidence of stroke and MACCE found in our study. The risk of CHD was also significantly increased in CKD stage 3 in our data. In fact, there is a lack of evidence to support increased CHD in metformin users. The high risk of CHD with metformin use might be caused by lactic acidosis [39]. However, as aforementioned, the risk of lactic acidosis with metformin use is very low, and the risk has been reported not to increase even in CKD stage 5 [12-14]. One previous study reported that metformin use could delay the endothelial recovery of a drug-eluting stent via an AMPK-dependent pathway, which could cause stent thrombosis [40]. Poor glycemic control might be another cause of the increased CHD in metformin users in our study because the baseline level of fasting glucose in metformin users was significantly higher than that in non-users. Because this study was conducted using data from a health screening cohort, we could not examine other glycemic control parameters, such as glycated hemoglobin. However, the low risk of mortality and ESRD become difficult to explain if metformin users had poor glycemic control during the follow-up period. 
Despite the increased risk of MAACE, metformin users had a significantly lower risk of cardiovascular mortality than non-users in our study. Interestingly, this paradoxical result was also reported in a previous study. A post hoc analysis of a randomized controlled trial reported that patients with metformin exposure had significantly lower risks of all-cause and cardiovascular mortality during follow-up than those without exposure [41]. However, the risk of stroke did not differ between groups (HR, 0.97; 95\% CI, 0.68-1.39), and the risk of MI was marginally increased in patients with metformin exposure (HR, 1.23; 95\% CI, 0.92-1.65). It is difficult to find evidence to support that contradictory result. As aforementioned, although AMPK activation caused by metformin can be harmful in patients with CKD, the AMPK pathway is also a well-known underlying protective mechanism for CVD [42]. Moreover, the role of AMPK in angiogenesis is known to be contradictory. Metformin suppresses retinal angiogenesis and exerts a protective effect against retinopathy [43], and it also suppresses tumor angiogenesis [44]. On the other hand, metformin promotes angiogenesis and revascularization under hypoxic and ischemic injury [45]. Thus, AMPK activation by metformin could have different angiogenic effects in different cellular microenvironments [46], which could affect its role in the development of CVD. Moreover, the metformin users in our study were younger and had fewer comorbidities and higher eGFR than the non-users. Therefore, metformin users might have survived CVD better than non-users. In addition, because we defined our outcomes based on diagnostic codes, not medical records, our operational definitions of the outcomes might not adequately reflect real clinical events, though hospitalization with a diagnostic code is a widely used method to define outcomes in big data studies $[13,16]$.

Metformin users had a significantly lower risk of allcause mortality during follow-up than non-users did in our study, which is inconsistent with previous studies $[15,19]$. This result might reflect the lower comorbidity levels of metformin users. However, survival benefits remained when we matched comorbidities in the PSM analysis. It is noteworthy that the survival benefit of metformin use was attenuated in patients at an advanced CKD stage in our sensitivity analysis. In a study by Hung et al. [13], metformin use was associated with an increased risk of allcause mortality during follow-up in patients with CKD stage 5 . They also reported that the mortality risk increased dose-dependently with metformin use in that population. Therefore, renal function depletion and consequent metformin accumulation might not be beneficial for mortality at an advanced CKD stage. Nevertheless, that study also reported a significantly decreased risk of ESRD in CKD stage 5. In our study, the decreased risk of ESRD conferred by metformin use was observed across all CKD stages. Recent experimental studies demonstrated that metformin prevented renal fibrosis and retarded CKD progression in murine models $[47,48]$. Nevertheless, because metformin use also conferred an increased risk of MACCE in our study, further experimental studies are needed to reveal the effects of metformin on other organs, beyond its protective effects on the kidney.

This study has several limitations. First, because we defined metformin use as 90 days of use before the index date, we could not consider metformin use before the health screening day. Thus, the actual duration of metformin use could differ from that used in the analysis. Second, we could not consider newly added, switched, or discontinued anti-diabetes medications (other than metformin) during the follow-up period. Therefore, the effects of other anti-diabetes medications might confound our findings. Third, we could not include the dose of metformin in the analysis. Thus, dose-dependent relationships between metformin and clinical outcomes were not presented. Fourth, although we conducted PSM analyses, hidden confounding factors might still have affected the relationship between metformin use and clinical outcomes. Fifth, some health screening centers might not have used isotope dilution mass spectrometry-traceable creatinine measurements. Thus, the possibility of CKD stage misclassification exists in this study. Finally, the study population in this work was Korean, and our results might not be generalizable to other ethnic groups. Despite those limitations, our study also has several strengths. Because this was a nationwide cohort study, a large number of patients were included. In addition, the NHIS database includes claims for all medical facilities in Korea, and data from Statistics Korea include all cases of mortality. Therefore, few outcomes in the data were missing. Moreover, we conducted sensitivity analyses for subgroups with PDC of $\geq 80 \%$ and found consistent relationships between metformin use and clinical outcomes. Thus, our results were not confounded by medication discontinuation. 
In conclusion, despite the current trend to expand metformin use in patients with CKD, we found that metformin use could be associated with an increased risk of MACCE in this population. On the other hand, metformin users had a lower risk of all-cause mortality and ESRD during follow-up than non-users did. Therefore, metformin needs to be used carefully under strict surveillance for CVD occurrence in patients with CKD.

\section{Conflicts of interest}

All authors have no conflicts of interest to declare.

\section{Funding}

This study was supported by a Young Investigator Research Grant from the Korean Society of Nephrology (2019), a National Research Foundation of Korea (NRF) grant funded by the Korean government (MSIT) (2019R1G1A1100498), and the Soonchunhyang University Research Fund.

\section{Authors' contributions}

\section{Conceptualization: HN, DRR}

Data curation: MHK

Formal analysis: MHK

Funding acquisition: $\mathrm{HK}$

Writing-original draft: HJO, SHK, JSJ, HN, DCH, HK, DRR

Writing-review \& editing: SHK, JSJ, HN, DCH

All authors read and approved final manuscript.

\section{ORCID}

Min Ho Kim, https://orcid.org/0000-0003-4909-2308 Hyung Jung Oh, https://orcid.org/0000-0002-4281-696X Soon Hyo Kwon, https://orcid.org/0000-0002-4114-4196 Jin Seok Jeon, https://orcid.org/0000-0003-2421-2289 Hyunjin Noh, https://orcid.org/0000-0002-1904-1684 Dong Cheol Han, https://orcid.org/0000-0002-8835-8642 Hyoungnae Kim, https://orcid.org/0000-0002-5359-0214 Dong-Ryeol Ryu, https://orcid.org/0000-0002-5309-7606

\section{References}

1. Zhang QL, Rothenbacher D. Prevalence of chronic kidney dis- ease in population-based studies: systematic review. BMC Public Health 2008;8:117.

2. Thomas B, Matsushita K, Abate KH, et al. Global cardiovascular and renal outcomes of reduced GFR. J Am Soc Nephrol 2017;28:2167-2179.

3. Vanholder R, Annemans L, Brown E, et al. Reducing the costs of chronic kidney disease while delivering quality health care: a call to action. Nat Rev Nephrol 2017;13:393-409.

4. Jha V, Garcia-Garcia G, Iseki K, et al. Chronic kidney disease: global dimension and perspectives. Lancet 2013;382:260-272.

5. Shaw JE, Sicree RA, Zimmet PZ. Global estimates of the prevalence of diabetes for 2010 and 2030. Diabetes Res Clin Pract 2010;87:4-14

6. Gansevoort RT, Correa-Rotter R, Hemmelgarn BR, et al. Chronic kidney disease and cardiovascular risk: epidemiology, mechanisms, and prevention. Lancet 2013;382:339-352.

7. Afkarian M, Sachs MC, Kestenbaum B, et al. Kidney disease and increased mortality risk in type 2 diabetes. J Am Soc Nephrol 2013;24:302-308.

8. Zelniker TA, Wiviott SD, Raz I, et al. SGLT2 inhibitors for primary and secondary prevention of cardiovascular and renal outcomes in type 2 diabetes: a systematic review and meta-analysis of cardiovascular outcome trials. Lancet 2019;393:31-39.

9. Bethel MA, Patel RA, Merrill P, et al. Cardiovascular outcomes with glucagon-like peptide-1 receptor agonists in patients with type 2 diabetes: a meta-analysis. Lancet Diabetes Endocrinol 2018;6:105-113.

10. Kim MK, Ko SH, Kim BY, et al. 2019 Clinical practice guidelines for type 2 diabetes mellitus in Korea. Diabetes Metab J 2019;43:398-406.

11. Davies MJ, D'Alessio DA, Fradkin J, et al. Management of hyperglycemia in type 2 diabetes, 2018: a consensus report by the American Diabetes Association (ADA) and the European Association for the Study of Diabetes (EASD). Diabetes Care 2018;41:2669-2701.

12. Salpeter SR, Greyber E, Pasternak GA, Salpeter EE. Risk of fatal and nonfatal lactic acidosis with metformin use in type 2 diabetes mellitus. Cochrane Database Syst Rev 2010;2010:CD002967.

13. Hung SC, Chang YK, Liu JS, et al. Metformin use and mortality in patients with advanced chronic kidney disease: national, retrospective, observational, cohort study. Lancet Diabetes Endocrinol 2015;3:605-614.

14. Inzucchi SE, Lipska KJ, Mayo H, Bailey CJ, McGuire DK. Metformin in patients with type 2 diabetes and kidney disease: a systematic review. JAMA 2014;312:2668-2675. 
15. Charytan DM, Solomon SD, Ivanovich $P$, et al. Metformin use and cardiovascular events in patients with type 2 diabetes and chronic kidney disease. Diabetes Obes Metab 2019;21:1199-1208.

16. Roumie CL, Chipman J, Min JY, et al. Association of treatment with metformin vs sulfonylurea with major adverse cardiovascular events among patients with diabetes and reduced kidney function. JAMA 2019;322:1167-1177.

17. Ekström N, Schiöler L, Svensson AM, et al. Effectiveness and safety of metformin in 51675 patients with type 2 diabetes and different levels of renal function: a cohort study from the Swedish National Diabetes Register. BMJ Open 2012;2:e001076.

18. Crowley MJ, Diamantidis CJ, McDuffie JR, et al. Clinical outcomes of metformin use in populations with chronic kidney disease, congestive heart failure, or chronic liver disease: a systematic review. Ann Intern Med 2017;166:191-200.

19. Kwon S, Kim YC, Park JY, et al. The long-term effects of metformin on patients with type 2 diabetic kidney disease. Diabetes Care 2020;43:948-955.

20. Lee MC, Lee CH, Chang LY, et al. Association of metformin use with end-stage renal disease in patients with type 2 diabetes mellitus: a nationwide cohort study under the pay-for-performance program. J Clin Pharmacol 2019;59:1443-1452.

21. Cheol Seong S, Kim YY, Khang YH, et al. Data Resource Profile: The National Health Information Database of the National Health Insurance Service in South Korea. Int J Epidemiol 2017; 46:799-800.

22. Ko MJ, Park CM, Kim YJ, Kang SH, Park DW. Clinical application and potential effects of 2014 hypertension guidelines on incident cardiovascular events. Am Heart J 2015;170:1042-1049.

23. Levey AS, Stevens LA, Schmid CH, et al. A new equation to estimate glomerular filtration rate. Ann Intern Med 2009;150:604612.

24. Sundararajan V, Henderson T, Perry C, Muggivan A, Quan H, Ghali WA. New ICD-10 version of the Charlson comorbidity index predicted in-hospital mortality. J Clin Epidemiol 2004;57:12881294.

25. Raebel MA, Schmittdiel J, Karter AJ, Konieczny JL, Steiner JF. Standardizing terminology and definitions of medication adherence and persistence in research employing electronic databases. Med Care 2013;51(8 Suppl 3):S11-21.

26. Hur KY, Kim MK, Ko SH, et al. Metformin treatment for patients with diabetes and chronic kidney disease: a Korean Diabetes Association and Korean Society of Nephrology consensus statement. Kidney Res Clin Pract 2020;39:32-39.

27. DeFronzo RA, Goodman AM. Efficacy of metformin in patients with non-insulin-dependent diabetes mellitus. The Multicenter Metformin Study Group. N Engl J Med 1995;333:541-549.

28. Chan JC, Tomlinson B, Critchley JA, Cockram CS, Walden RJ. Metabolic and hemodynamic effects of metformin and glibenclamide in normotensive NIDDM patients. Diabetes Care 1993;16:1035-1038.

29. Boussageon R, Supper I, Bejan-Angoulvant T, et al. Reappraisal of metformin efficacy in the treatment of type 2 diabetes: a meta-analysis of randomised controlled trials. PLoS Med 2012;9: e1001204.

30. Lamanna C, Monami M, Marchionni N, Mannucci E. Effect of metformin on cardiovascular events and mortality: a meta-analysis of randomized clinical trials. Diabetes Obes Metab 2011;13:221-228.

31. Griffin SJ, Leaver JK, Irving GJ. Impact of metformin on cardiovascular disease: a meta-analysis of randomised trials among people with type 2 diabetes. Diabetologia 2017;60:1620-1629.

32. Ong CR, Molyneaux LM, Constantino MI, Twigg SM, Yue DK. Long-term efficacy of metformin therapy in nonobese individuals with type 2 diabetes. Diabetes Care 2006;29:2361-2364.

33. He L, Wondisford FE. Metformin action: concentrations matter. Cell Metab 2015;21:159-162.

34. McCullough LD, Zeng Z, Li H, Landree LE, McFadden J, Ronnett GV. Pharmacological inhibition of AMP-activated protein kinase provides neuroprotection in stroke. J Biol Chem 2005;280:2049320502.

35. Jia J, Cheng J, Ni J, Zhen X. Neuropharmacological actions of metformin in stroke. Curr Neuropharmacol 2015;13:389-394.

36. Kim HI, Lee JS, Kwak BK, et al. Metformin ameliorates lipotoxic $\beta$-cell dysfunction through a concentration-dependent dual mechanism of action. Diabetes Metab J 2019;43:854-866.

37. Lalau JD, Kajbaf F, Bennis Y, Hurtel-Lemaire AS, Belpaire F, De Broe ME. Metformin treatment in patients with type 2 diabetes and chronic kidney disease stages 3A, 3B, or 4. Diabetes Care 2018;41:547-553.

38. Chien LN, Chou CL, Chen HH, et al. Association between stroke risk and metformin use in hemodialysis patients with diabetes mellitus: a nested case-control study. J Am Heart Assoc 2017;6:e007611.

39. Kendrick J, Shah P, Andrews E, et al. Effect of treatment of metabolic acidosis on vascular endothelial function in patients with CKD: a pilot randomized cross-over study. Clin J Am Soc Nephrol 2018;13:1463-1470.

40. Habib A, Karmali V, Polavarapu R, et al. Metformin impairs vascular endothelial recovery after stent placement in the setting of 
locally eluted mammalian target of rapamycin inhibitors via S6 kinase-dependent inhibition of cell proliferation. J Am Coll Cardiol 2013;61:971-980.

41. Bergmark BA, Bhatt DL, McGuire DK, et al. Metformin use and clinical outcomes among patients with diabetes mellitus with or without heart failure or kidney dysfunction: observations from the SAVOR-TIMI 53 trial. Circulation 2019;140:1004-1014.

42. Shirwany NA, Zou MH. AMPK in cardiovascular health and disease. Acta Pharmacol Sin 2010;31:1075-1084.

43. Han J, Li Y, Liu X, et al. Metformin suppresses retinal angiogenesis and inflammation in vitro and in vivo. PLoS One 2018;13: e0193031.

44. Wang J, Li G, Wang Y, et al. Suppression of tumor angiogenesis by metformin treatment via a mechanism linked to targeting of
HER2/HIF-1a/VEGF secretion axis. Oncotarget 2015;6:4457944592.

45. Calvert JW, Gundewar S, Jha S, et al. Acute metformin therapy confers cardioprotection against myocardial infarction via AMPK-eNOS-mediated signaling. Diabetes 2008;57:696-705.

46. Li Y, Sun R, Zou J, Ying Y, Luo Z. Dual roles of the AMP-activated protein kinase pathway in angiogenesis. Cells 2019;8:752.

47. Neven E, Vervaet B, Brand K, et al. Metformin prevents the development of severe chronic kidney disease and its associated mineral and bone disorder. Kidney Int 2018;94:102-113.

48. Lee M, Katerelos M, Gleich K, et al. Phosphorylation of acetyl-CoA carboxylase by AMPK reduces renal fibrosis and is essential for the anti-fibrotic effect of metformin. J Am Soc Nephrol 2018;29:2326-2336. 